\title{
PROGRAMA TUTORIAL COMPLEMENTO PARA LA EDUCACIÓN INTEGRAL
}

\author{
Juan Carlos Torres Sandoval * Wilson Joven Sarria** Jairo Jamith Palacios Rozo**
}

\section{RESUMEN}

La institucionalización de los programas tutoriales al interior de los entes educativos es importante, pues con ello se busca un aporte significativo para la educación integral de sus educandos. Para diseñar uno adecuado, se debe realizar el levantamiento de las necesidades de los estudiantes; además, se ha de tener presente que ellos se transforman a lo largo de la carrera, en cuyo proceso van madurando. Por consiguiente, se requiere identificar las etapas precisas de tales cambios, a fin de que el modelo que se diseñe sea el más acorde. Por otra parte, Se hace indispensable también acudir a herramientas tecnológicas que permitan lograr un aspecto de transparencia en la aplicación del modelo para toda institución, procurando, de esta manera, extenderlo a todas las actividades en las que participa cada uno de los miembros de la comunidad educativa.

\section{PALABRAS CLAVE}

Acción tutorial, Tecnologías de la Información y la Comunicación, TIC, Virtualidad.

\begin{abstract}
It is important to institutionalize tutorial programs inside the educational institutions, looking for a significant contribution for the integral education of students. For the design, it is necessary to find the students' needs, it is also necessary to understand that students grow up during the process. For this reason, it is necessary to identify the stages according to those changes, so the model to design would be the one that fits the best. It is also necessary to use technological tools to get a transparent aspect for the application of the model for any institution, in such a way the model can be extended to all the activities that each one of its members participates in.
\end{abstract}

\section{KEYWORDS}

Tutorial Action, Information and Comunication Technologies TIC, Virtuality.

\footnotetext{
* Magíster y Doctorando en Pedagogía, Universidad Popular Autónoma del Estado de Puebla - UPAEP. Profesor Tiempo Completo, Fundación Universitaria Empresarial de la Cámara de Comercio de Bogotá. UNIEMPRESARIAL.jktorres2003@yahoo.com.

** Magister en Ciencias de la información y las comunicaciones, Universidad Distrital Francisco José de Caldas, Profesor Universidad de Cundinamarca.wjoven@gmail.com

*** Magister en Educación, Universidad Santo Tomás, Profesor Tiempo Completo, Universidad ECCI. Palacios.jairo@gmail.com
} 


\section{INTRODUCCIÓN}

En la actualidad, el modelo tutorial cobra cada vez mayor importancia para la labor pedagógica, en tanto una respuesta de calidad del sistema educativo hacia la sociedad. "apostar por un sistema de acción tutorial universitario es una forma de dar respuesta a la creciente exigencia social sobre los rendimientos y resultados del sistema universitario público" (CARDOZO ORTIZ, 2010); con ello se contribuye a la educación integral de los estudiantes. Por otra parte, las TIC son el motor que hoy mueve al mundo. Poder mezclar estas dos corrientes en un mismo objeto de estudio genera una gran expectativa para el futuro de la educación.

La modalidad de estudio actual demanda trabajo autónomo del estudiante. El espacio creado por él debe tenerse presente, de manera que su labor académica habitual no se descontextualice con el trabajo desarrollado por medio del modelo pedagógico institucional. En consecuencia, allí es donde se debe concentrar el foco de la tutoría, que para este caso adquiere la forma virtual, necesaria para complementar la educación integral del alumno. Por tal razón, es transcendental ampliar los límites de la tutoría tradicional (presencial) hacia la virtualidad, de tal modo que se trate de atender las necesidades de los estudiantes presenciales y, al mismo tiempo, ellos puedan desarrollar su trabajo autónomo.

No hay que olvidar que la formación de los individuos no solo se produce a temprana edad sino que está presente en todos los momentos de la vida. El ideal del desarrollo humano está basado en el cambio permanente de forma positiva. Normalmente, durante las primeras etapas de la vida, se observa acompañamiento de las personas, pero en el momento de pasar a estudios superiores esto no ocurre con frecuencia. Por tal razón, es evidente la necesidad de la acción tutorial como respuesta a la falencia que se detecta al interior de las instituciones de educación.

Ahora bien, conviene resaltar que aunque la educación universitaria por excelencia está desarrollada hacia una profesión específica, es decir, que su enfoque disciplinar es hacia las temáticas propias de la carrera, en la actualidad se demanda un enfoque holístico que mejore la calidad de vida del estudiante y favorezca su formación integral. Ante este hecho, las entidades de educación superior se preocupan por involucrar actividades que fortalezcan tales aspectos. Hasta ahora, el papel tradicional que ha tenido el docente ha sido pasivo, se ha limitado a impartir su conocimiento sin involucrarse más de lo necesario con sus alumnos, sin darse cuenta de la necesidad de avanzar, de encontrar nuevos retos.

A lo anterior se suma el débil contacto de los discentes con sus docentes en su trabajo autónomo, por lo cual se hace necesario un modelo de intervención que garantice el aprendizaje significativo tanto en el acompañamiento directo en aula como durante su trabajo independiente. En el momento de diseñar un modelo tutorial a la medida de cada una de las instituciones, se pretende garantizar el acompañamiento de los alumnos en todas sus instancias, con el fin de proveer una educación integral.

\section{Fundamentación}

1.1 Justificación: La tutoría, como complemento del quehacer del maestro, se está implementando a nivel mundial. "posiblemente estemos viviendo una revolución en la creación de conocimiento más fuerte que la revolución industrial o los profundos cambios producidos en nuestra cultura con la invención 
de la imprenta o la televisión" (FERNANDEZ VARA, 2014), lo que convierte a las TIC en el complemento ideal para la acción tutorial propuesta.

El sentido real del profesor cobra relevancia cuando complementa la acción pedagógica, normalmente desarrollada al interior de la clase, con su tarea tutorial. El "deber ser" de un maestro no solo es impartir conocimientos, él adquiere un compromiso ético con el estudiante, con la sociedad y consigo mismo, al momento de recibir en el aula a sus nuevos educandos. Él debe ser consciente de su llamado a seguir formando a ese joven, no solo en la ciencia sino también en su esencia. Por ello, el docente necesita, adicionalmente, desarrollar su competencia en la tutoría, dado que es por medio de esta que puede lograr darle pleno significado a su labor pedagógica.

El modelo tutorial en Colombia es reciente. Hasta este momento se relaciona directamente con el asesoramiento que se le presta al estudiante en sus debilidades académicas, sin tener en cuenta otro tipo de dimensiones significativas para su desarrollo integral. La modalidad educativa apoya al alumno en su adaptabilidad al entorno de la institución, se crean ambientes agradables de trabajo y la atención al discente es de forma remedial, por medio de bienestar institucional, pero no va más allá, no se indaga sobre el entorno en el que vive ni un manejo preventivo de sus diferentes problemáticas. Allí las necesidades del educando se evidencian por el poco contacto institucional con él, con su trabajo autónomo,

A este respecto, el Ministerio de Educación de Colombia, en su informe de rendición de cuentas de octubre de 2012 a noviembre de 2013, afirma que uno de sus retos es bajar la deserción universitaria anual del $12,9 \%$ en el 2010 al $9 \%$ en el 2014. La apuesta por un programa tutorial va muy de la mano con la solución a esta problemática, que actualmente afecta a todas las universidades. Muchos de los inconvenientes que presentan los estudiantes no son conocidos por las instituciones. Alli se ve, pues, la necesidad de los tutores a fin de que orienten al alumno en sus diferentes facetas, le den la tranquilidad y seguridad para afrontar sus diversas dificultades e incluso recibir consejos. Muchos de los problemas de los jóvenes asociados a la deserción podrían tener una solución práctica si fueren conocidos por los tutores en el tiempo pertinente.

1.2 Problemática: En la actualidad se manifiesta una tendencia hacia la formación con una educación integral. Definitivamente se cambió el concepto del aprendizaje teórico como única opción posible para la educación, ahora es importante también el entorno en el que el estudiante aprende y no solo el hecho de llenarse de una serie de conceptos. Los diferentes contextos en los que se encuentra el educando son esenciales para su formación. Así, la tutoría se convierte en una estrategia fundamental para interactuar con el alumno y su entorno próximo.

En tales condiciones, es indispensable contar con la información de los diferentes actores para la elaboración de un programa tutorial destinado a las instituciones, de manera que el diseño del mismo esté directamente relacionado con la problemática que se pueda detectar en los estudiantes, de forma que, a partir de esta, se pueda orientar el programa tutorial de manera adecuada.

\section{Estado Del Arte}

2.1 Orientación educativa: El orientador es considerado como el motor de la innovación educativa y, en tal sentido, se le demandan competencias difíciles de adquirir para su labor, las cuales deben desarrollarse sin el apoyo de un equipo de trabajo como lo son los 
docentes. Así las cosas, es indispensable hacer claridad en que el trabajo de orientación educativa al interior de la institución compete al orientador, quien con su labor debe generar una cultura institucional al respecto, actividad desplegada, principalmente, por los docentes en todos los ámbitos educativos.

Los ámbitos disciplinares de la orientación psicopedagógica están enfocados hacia la orientación vocacional, a la educación especial, a la psicología de la educación y a la didáctica. La orientación se empezó a desarrollar en Estados Unidos a principios del siglo $X X$. Los movimientos que lograron este nacimiento fueron varios: se destaca el trabajo de Parsons, quien tuvo un enfoque principal hacia la orientación vocacional.

Los factores comunes encontrados son: el movimiento reivindicativo de reformas sociales tras la revolución industrial, el movimiento psicométrico, el modelo de orientación basado en la teoría de rasgos y factores del movimiento americano por la salud mental y el counseling, y las diferentes organizaciones profesionales de orientación tanto en Estados Unidos como en Europa, principalmente en Francia.

El proceso de la evolución de la orientación, presentado en los párrafos anteriores, está encaminado hacia la educación, entendiéndola como algo más que instruir, donde el equipo docente tiene una participación activa en el mismo y donde se evidencia que dicho proceso debe estar presente en todas las etapas de la formación, además de todas las clases o actividades. En ese contexto, existen muchas definiciones de orientación educativa, de acuerdo con cada autor.

Conjunto de conocimientos, metodologías y principios teóricos que fundamentan la planificación, diseño, aplicación y evaluación de la intervención psicopedagógica preventiva, comprensiva, sistémi- ca y continuada que se dirige a las personas, las instituciones y el contexto comunitario, con el objetivo de facilitar y promover el desarrollo integral de los sujetos a lo largo de las distintas etapas de su vida, con la implicación de los diferentes agentes educativos (orientadores, tutores, profesores, familia) y sociales.

2.2 Educación integral y formación docente: Como complemento a los conceptos principales de tutoría y virtualidad se contemplan los de Educación Integral y Formación Docente que los apoyan directamente. El fin último de cualquier contexto educativo debería ser la integralidad de la educación, en donde no solo se crece intelectualmente sino como persona dentro de una sociedad. Para que se pueda dar esta situación se necesita que los profesores tomen conciencia de su papel protagónico y sepan cómo actuar acorde con la situación a la que se ven expuestos. El comportamiento que se espera, demanda que los maestros participen en una capacitación permanente que les entregue las herramientas adecuadas para este contexto.

Según la revisión bibliográfica, se evidencia que en la última década, en cuanto a tutoría, es uno de los mayores exponentes de este tipo de enfoque. Además, ha de considerarse también el trabajo realizado por Riart a fin de lograr establecer una base teórica robusta a partir de estos dos autores.

Ahora bien, el tema de la tutoría es un componente relevante, pero también es de especial cuidado el trabajo a realizar con el enfoque virtual de la misma. Definitivamente se hace necesario determinar el punto de encuentro de estos dos temas para su buen desarrollo. Con el propósito de ubicarlo, se inicia con una revisión de los argumentos principales que exponen tanto Bisquerra como Riart en cuanto a tutoría, diferenciándolos de otros autores que también 
han trabajado los mismos conceptos; luego se referencian los aportes hacia la virtualidad y finalmente cómo se relacionan estos dos.

El análisis del contexto arroja un diagnóstico sobre el que se puede evidenciar si existen o no necesidades en el entorno examinado. Además, a partir del diagnóstico se puede definir el enfoque del modelo de intervención a realizar.

La identificación de necesidades permite, a su vez, reconocer a los actores involucrados en el contexto de orientación educativa y muestra las relaciones entre ellos. Es sustancial involucrar a estos actores en la intervención psicopedagógica a realizar, de forma que el modelo sea efectivo.

Bisquerra en particular es uno de los autores que mejor reflejan el trabajo tutorial; lo ha analizado desde diferentes puntos de vista, ha escrito varios libros en compañía de otros investigadores, lo cual genera una mayor consistencia en las teorías trabajadas. Tiene presente el entorno en el cual se produce el conocimiento, además de darle realce a la labor docente, involucrándola en una educación integral a partir de la acción tutorial.

Desde tiempos remotos, la tecnología ha acompañado al hombre, ayudándole a la satisfacción de sus necesidades. En las últimas décadas, su uso se ha enfocado hacia los jóvenes, con creaciones como la telefonía móvil, los videojuegos y la Internet. "Para los adolescentes, Internet es una herramienta importante para sus estudios y un elemento de diversión, información y relación" (GARCIA \& LOMBARTE, 2004). Las nuevas tecnologías proveen a los jóvenes de una nueva flexibilidad, independencia y privacidad en sus comunicaciones interpersonales, que se pueden acotar hacia una complementariedad en su educación integral.
Aún no hay estudios científicos que aclaren si las nuevas tecnologías tienen enfoques más negativos que positivos, todo se basa en opiniones; lo cierto es que "las Tecnologías de la Información (TI), particularmente aplicadas en el ámbito educativo, juegan un papel crucial en la conformación de actores innovadores, creativos y competitivos en el mundo global contemporáneo" (PADILLA, 2011)

\subsection{Tutoría como estrategia de apoyo en la educación}

superior: Tutoría son sustanciales los diferentes contextos en los que interactúa el estudiante. En tal sentido, la educación va más allá de lo escolar; plantea que también se considera educación a la formación continua en las empresas, en los medios de comunicación, en la familia, etc. Además, considera que todas estas situaciones pueden requerir algún tipo de orientación psicopedagógica. Por tanto, se deben identificar los diferentes entornos en los que se desenvuelven los alumnos, indagar cuáles son las necesidades más sentidas de cada uno de ellos y, con base en esos resultados, desarrollar el modelo de intervención adecuado a la realidad que vive el discente.

Bajo esas consideraciones, varios autores proponen diferentes definiciones sobre la tutoría:

[...] como una acción orientadora global, clave para aglutinar tanto los aspectos académicos como los educativos, el tutor se contempla como un elemento necesario para el escolar, el grupo de alumnos, para la relación con la familia y con el contexto social (VALLE LOPEZ, 2004).

Por tanto el modelo tutorial debe tener en cuenta el sentido de la diversidad, aunque sin dejar de lado que hoy por hoy está cobrando fuerza otro tipo de terminología que va más allá: la singularidad.

Es consciente de esto y lo expresa diciendo que cuan- 
do se habla de diversidad se entiende que existen estudiantes dentro del grupo que tienen diferencias con sus compañeros, pero que definitivamente las acciones a realizar deben tener en cuenta a la gran mayoría. La singularidad considera a cada uno de los participantes del proceso tanto a nivel individual como de grupo y trata al extremo de dar soluciones para cada uno de los distintos casos particulares.

\subsection{Tutoría virtual: una estrategia de apoyo al es-} tudiante desde la distancia: Uno de los conceptos fuertes en este estudio es el de la virtualidad, dado que es necesario contemplar todas las posibles herramientas para poder atender satisfactoriamente a los estudiantes. Es así como la virtualidad juega un papel primordial al intentar tener presentes a todos los alumnos, sobre todo cuando no se encuentran en el campus de la institución.

El concepto de virtualidad tiene diferentes acepciones. Virtualidad indica irrealidad, aunque en el caso de las Tecnologías de la Información y la Comunicación (TIC), más que irrealidad sería inmaterialidad, que es otra acepción del concepto. En definitiva aquello que está presente pero que es intangible, que no podemos tocar.

La Tecnología Educativa aborda el proceso enseñanza-aprendizaje en forma sistemática y organizada, y nos proporciona estrategias, procedimientos y medios emanados de los conocimientos científicos en que se sustenta. [Está enfocada a solucionar] tres problemas básicos, entre los muchos que la obstaculizan:

1. Educar a un número cada vez mayor de personas.

2. Educar mejor y con mayor eficacia.

3. Educar más con menos costo.
El autor hace también alusión a algo que es verdaderamente preocupante: todos los sectores cambian acorde con la necesidad de acomodarse a los nuevos tiempos, menos la educación. Ha llegado el momento en que se debe realizar su transformación.

La aplicación de una ciencia a la solución de problemas prácticos, y de allí también se logra definir el rol del tecnólogo educativo así: lo que el tecnólogo educativo sabe es que su función crucial como educador radica en que estructura el medio ambiente a favor de la educación y que este proceso de estructuración educacional o instruccional del medio es la tecnología, a través de la cual, aspectos reproducibles del arte de educar pueden ser analizados efectivamente, así como imitado

\section{CONCLUSIONES}

En consecuencia, la educación integral, buscada desde la tutoría, no debe dejar atrás el uso de las nuevas tecnologías como la virtualidad, sino más bien sacar el mejor provecho de esta, considerando que es el punto más cercano a los jóvenes actuales.

El planteamiento está encaminado a identificar las problemáticas de los estudiantes en trabajo independiente, teniendo como base la virtualidad. Es conveniente que las universidades garanticen que su modelo pedagógico permee a la totalidad de sus alumnos, por lo cual es importante que la tutoría se apoye en las TIC.

Se necesita, de una transformación en el paradigma actual de atención a las necesidades de los jóvenes. Todo cambio trae consigo complicaciones.

La tecnología trae expectativas pero también incertidumbres que pocos están dispuestos a reconocer. 


\section{REFERENCIAS}

CARDOZO ORTIZ, C. E. (2010). Reconstruccion de realidades, sueños y vidas desde uan mirada colectiva. Bucaramanga: Universidad Industrial de Santander.

FERNANDEZ VARA, G. (24 de Noviembre de 2014). Posiblemente estemos en el mes de noviembre más duro de la crisis. Periodico Extramadura.

GARCIA, I., \& LOMBARTE, S. (2004). Estudio de un caso sobre el uso de las tecnologías de la información y la comunicación para la inclusión Social. Barcelona: Universidad de Barcelona.
PADILLA, G. B. (2011). Las Tecnologías de la Información y el Conocimiento. La prensa.

VALLE LOPEZ, A. d. (2004). La tutoria integrada a la vida de un estudiante. En Contextos educativos y accion tutorial (pág. 9). Madrid: Ministerio de Educación, cultura y deporte. 